\title{
Outline of a System for Integrated Adaptive Ice Tracking and Multi-Agent Path Planning
}

J onatan Olofsson, Clas Veibäck, Gustaf Hendeby and Tor Arne J ohansen

\section{Conference article}

N.B.: When citing this work, cite the original article.

Part of: Proceedings of the 2017 Workshop on Research, Education and Development of Unmanned Aerial Systems (RED-UAS) 2017, pp.13-18 . ISBN: 978-1-5386-0939-2 (electronic), 978-1-5386-0940-8 (print)

DOI: https:// doi.org/ 10.1109/RED-UAS.2017.8101636

\section{Copyright:}

(C2017 IEEE. Personal use of this material is permitted. However, permission to reprint/republish this material for advertising or promotional purposes or for creating new collective works for resale or redistribution to servers or lists, or to reuse any copyrighted component of this work in other works must be obtained from the IEEE.

Available at: Linköping University Institutional Repository (DiVA)

http:// urn.kb.se/ resolve?urn=urn:nbn:se:liu:diva-141910 


\title{
Outline of a System for Integrated Adaptive Ice Tracking and Multi-Agent Path Planning
}

\author{
Jonatan Olofsson*, Clas Veibäck ${ }^{\dagger}$, Gustaf Hendeby ${ }^{\dagger}$, Tor Arne Johansen* \\ * Center for Autonomous Marine Operations and Systems (NTNU-AMOS) \\ Department of Engineering Cybernetics, \\ Norwegian University of Science and Technology, Trondheim, Norway. \\ jonatan.olofsson@ntnu.no, tor.arne.johansen@ntnu.no \\ $\dagger$ Department of Automatic Control, \\ Linköping University, Linköping, Sweden. \\ clas.veiback@liu.se, gustaf.hendeby@liu.se
}

\begin{abstract}
In polar region operations, drift sea ice positioning and tracking is useful for both scientific and safety reasons. Modeling ice movements has proven difficult, not least due to the lack of information of currents and winds of high enough resolution. Thus, observations of drift ice is essential to an up-todate ice-tracking estimate.

Recent years have seen the rise of Unmanned Aerial Systems (UAS) as a platform for geoobservation, and so too for the tracking of sea ice. Being a mobile platform, the research on UAS path-planning is extensive and usually involves an objective-function to minimize. For the purpose of observation however, the objective-function typically changes as observations are made along the path.

Further, the general problem involves multiple UAS and — in the case of sea ice tracking — vast geographical areas.

In this paper we discuss the architectural outline of a system capable of fusing data from multiple sources UAS's and others - as well as incorporating that data for both path-planning, sea ice movement prediction and target initialization. The system contains tracking of sea ice objects, situation map logic and is expandable as discussed with path-planning capabilities for closing the loop of optimizing paths for information acquisition.
\end{abstract}

\section{INTRODUCTION}

Drift sea ice is a major limiting factor in regions with Arctic conditions, often restricting the operational season to a few months of the year [8]. Ice Management is the field encompassing all activities to reduce or avoid impact from ice features [4]. In general terms, this includes detection, tracking and forecasting of ice features but also the threat evaluation and the physical management of breaking or towing ice objects [4]. Traditionally, shipmounted radars and visual detection from e.g. manned flights [1] have been used to detect potential threats, to aid in the manual decision process to launch countermeasures [4, 22]. Also, in severely ice-plagued operations, icebreakers have used to pre-emptively break down large upstream icefloes to managable pieces [15].
Recent years have seen increased availability of new types of sensor platforms - autonomous unmanned systems under [Autonomous Underwater Vehicle, AUV] and over [Unmanned Aerial System, UAS] water [7] capable of aiding in the situation awareness of the operation. Not only is this new class of platforms capable of operating with greater availability than previous sensors, but potentially also at a reduced cost.

With a limited field-of-view, sensors rely on moving to cover larger areas. Whereas traditionally sensors can be attached to man-controlled vessels, the development of autonomy for these sensors involve the automated planning of new routes $[2,13]$ through which the carrier should follow autonomously to aquire relevant sensor data. As data is acquired, the planning relies on the feedback of findings into the consideration of route replanning. This has also been studied for single-UAS optimal control in e.g. [13]. In [13], the proposed framework is an optimal-control scheme generating UAS intermediatelevel guidance, whereas the proposed approach in this article is to provide high-level agent-generic paths which, for each agent, can be converted locally to low-level control signals. Further, ice tracking and path planning using occupancy grids have been explored in e.g. [6, 10].

In Figure 1, an outline is presented with the overview of a system for the continuous intergration of sensor input and situation awareness into the optimization problem of assigning sensor paths which maximize the utility of each sensor. As reflected in Figure 1, the general tracking problem formulation is that of multi-agent, multi-target tracking. On a sensor level, expanded upon in Section II, many senors of varying types can contribute to the full image of the situation.

In our proposed architecture, the data from each sensor is fed into a central controller — see Section III — which summarizes the information for both user presentation and optimization of paths for future data acquisition. For this purpose, we also discuss — in Section III-B — the 


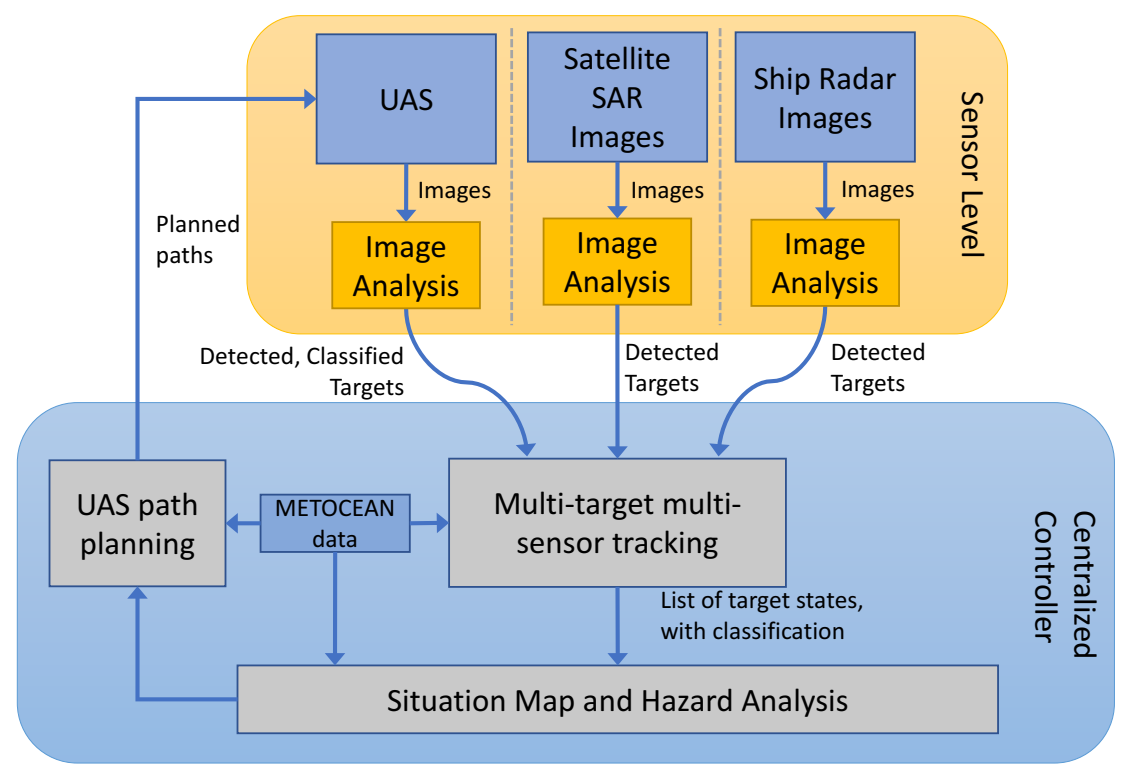

Figure 1: System overview

components available to form an optimization criterion to use in the problem of path planning. Partial results are presented in Section IV, followed by a discussion of past and expected results in Section V.

\section{Sensor Level}

A wide variety of sensors can be of use in the process of detecting and tracking sea ice, and a comprehensive summary can be found in [7]. Sensors with varying fieldof-views are, often without distinction, considered to be attached to a carrier - a movable sensor platform, such as a ship or a UAS. One way to categorize the sensors and sensor platforms is as

stationary sensors with known, fixed position and field-of-view, such as ground-based radar;

traceable sensors with known but not fixed position and field-of-view, albeit not controlable, such as satellites;

controllable sensors with known position and field-ofview, with the ability to be commanded to new locations, such as UAS's.

Whereas all above types of sensors are compatible with the system in Figure 1, the feedback from the sensors of the system can only be applied to controllable sensors.

Each sensor has different ways of measuring the presence of ice, and a sensor platform can be equipped with several types of sensors simultaneously. In the example of Figure 2, detections are extracted from infrared im- agery [14]. Each type of detection has unique characteristics in terms of e.g. noise and error covariance [5]. For the purpose of forming a generic situation awareness map, it is desirable to standardize the method of reporting measurements even though this will lead to loss of information e.g. due to coordinate transforms [5].

Other considerations for the sensor-controller interface involve the inclusion of other properties of the ice, such as - as available in imaging sensors - Hu moments [9] of each detection.

\section{Centralized Controller}

In the proposed architecture, from Figure 1, sensor reports are fed to a central controller, with responsibility for data fusion, situation map and high-level path planning.

\section{A. Data Fusion}

The first role of this controller is the fusing of the information from all sensor sources into a combined map of ice objects and ice coverage. The bounds of this map are not necessarily defined, but can span the entire globe with varying resolution. This means consideration has to be taken to the scalability of the map and to the algorithms employed. Sea ice coverage mapping can be performed using occupancy grids [14] whereas identifiable individual objects are tracked with sparse target tracking $[7,16,17,19]$. In the former case, scalability can be achieved through variable resolution, whereas in 


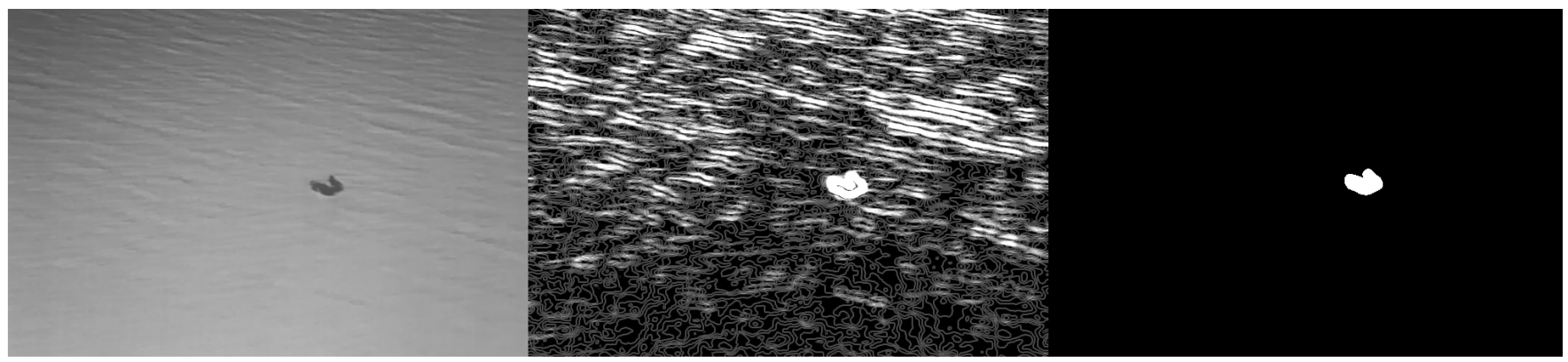

Figure 2: Detections of ice can be extracted from infrared imagery from UAS-carried cameras. Left: Raw thermal image; Middle: Enhanced features; Right: Segmented detected sea ice [14]. Image courtesy of Frederik S. Leira.

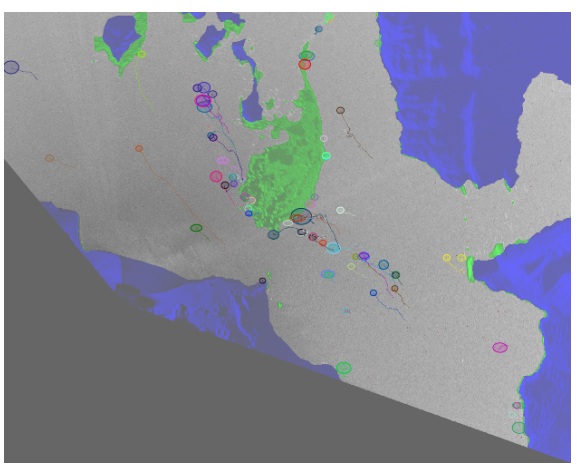

(a) Tracks after $2 \mathrm{~h}$.

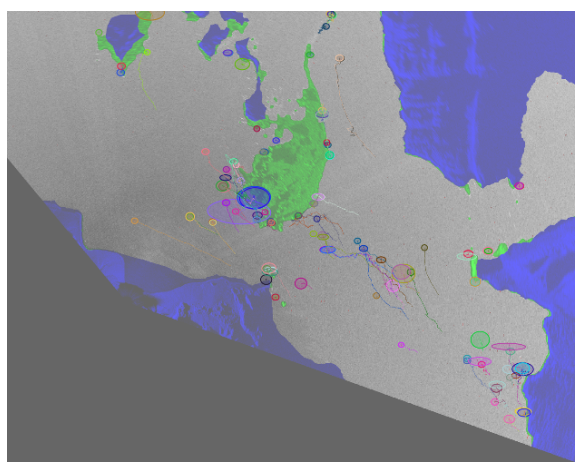

(b) Tracks after $4 \mathrm{~h}$.

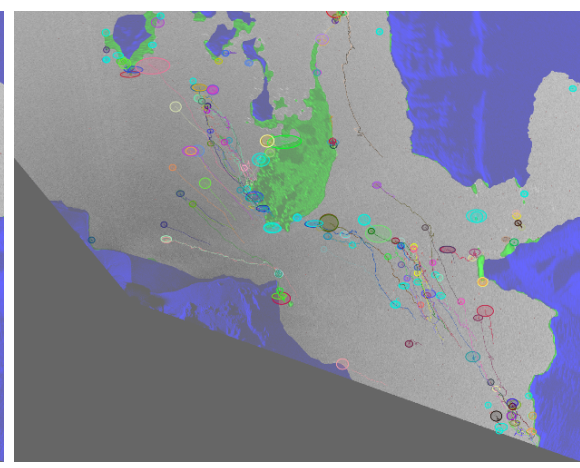

(c) Tracks after $7 \mathrm{~h}$.

Figure 3: Drift ice tracks over time, showing the land mask in blue and stationary detections in green. Tracks and targets retain an individual randomly assigned color over time.

the latter, objects can be stored in efficient datastructures $[16,17]$ to limit the number of objects involved in each update. An example result of the data fusion, from [19], is found in Figure 3.

Each choice of map coordinate system will have its advantages and disadvantages due to nonlinearities and discontinuities. As ice tracking is constrained to the water surface, our coordinate system of choice for global tracking is by latitude/longitude/altitude — LALoA. Since the problem is constrained to the sea surface, this naturally devolves to a two-dimensional latitude/longitude system.

\section{B. Situation Map}

Another role of the central controller is to form what we call a situation map. The map of tracked objects in Section III-A is one of the components of this map; other examples include

- sensor locations;

- traffic information;

- weather conditions; and

- information quality.

The main objective of the proposed system is to provide good situational awareness of where the relevant ice objects in the region are, as well as where there are no ice objects. To achieve this the objective function should reward paths resulting in large amounts of high-quality information.

The tracks from the centralized tracking algorithm naturally provide information on where the sea ice is as well as measurements of the quality of that information. This could directly be used to build an information quality map. However, a disadvantage of only considering the resulting tracks is that no information is available to discern whether an area is empty or simply unobserved a lack of detections in a region also provides information on the situation that should be incorporated into the map. One aspect that is handled by the tracking algorithm naturally is that information degrades over time. This approach was studied in [18] for the uncertainties of currents and winds from ice observations.

Another option is to bypass the information quality obtained from the tracking algorithm and directly consider the information obtained by the sensors. This is done in [20] where the information in each point over a grid is estimated using a large number of extended information filters. This can be rather costly for large highresolution grids, but naturally handles the information resulting from no detections. In this approach, forgetting of information over time needs to be handled explicitly. A relevant issue for both approaches is also discussed in [20] regarding the conversion of information on matrix form 


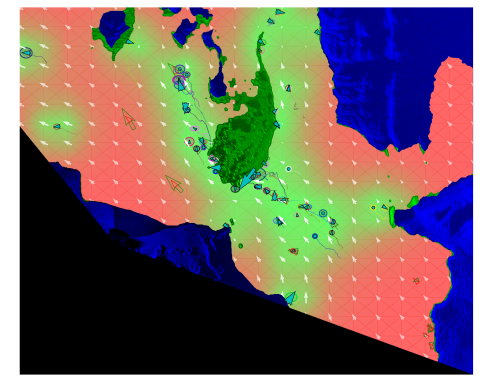

(a) Using the data from the MTT tracker, we use Gaussian fields to estimate both the velocity field in an image as well as its estimated model covariance (ranging from red (high) to green (low)).

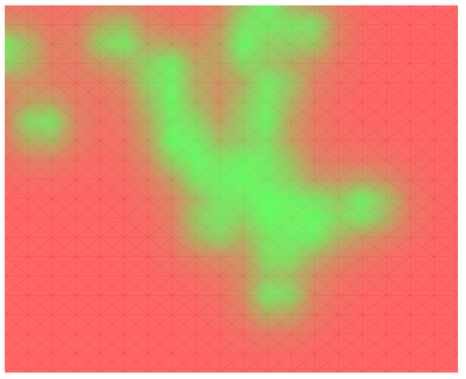

(b) The information map is determined by the trace of the Gaussian field velocity covariance in each point

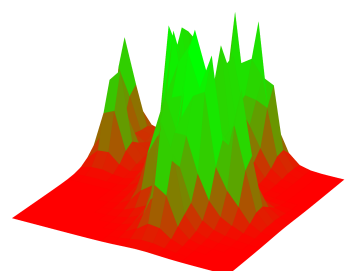

(c) The data in $4 \mathrm{~b}$ can also be interpreted as a $3 \mathrm{D}$ landscape to be navigated ( $\mathrm{z}$-axis is inverted).

Figure 4: The objective function used for path planning can be constructed from different information metrics depending on the optimization objective. One such objective is the determination of a resulting velocity-field from currents and wind.

to a comparable estimate on scalar form.

Weather and oceanic conditions (METOCEAN data) can be incorporated into the situation map in order to allow the motion planner to, for example, account for winds, and to avoid hazardous situations for the vehicles. In Arctic conditions, icing and severe winds is a real problem which need to be managed either through mitigation, as in e.g. [21] or the cancellation of flight operations.

In addition to the awareness of the physical properties in an area, the situation map should also take into account user priorities. This can be provided either by an operator or by some other source, such as traffic information. For example, it is more useful to have highquality information in regions with heavy traffic than in those in lack of human activity. Besides constructing the information used by the subsequent agent path planner, the same data can be used to create other situation maps, tuned for example for presentation to operators.

\section{Path Planning}

Since UASs are mobile sensor platforms they need to be controlled. Control of an aircraft is a multi-level control problem, and whereas low-level control is assumed to be handled locally by each agent, the situation awareness of the centralized controller can be used to provide each UAS with a path to follow. This path could in the simple case be a pre-determined route to scout along, but in the more advanced case, feedback control for the data acquisition is possible.

The main objective of the proposed system is to provide good situational awareness of the relevant ice objects in the region, as well as where there is open water. To achieve this the objective function should reward paths resulting in large amounts of high-quality information. An estimate of this quantity, given the path, can be obtained using the information quality map discussed in Section III-B.

Formulations and solutions to problems of this form are discussed in [20], including constraints such as dynamics of the vehicles and field of view. A concern with the solver algorithm used in [20], namely receding horizon, is that it quickly becomes very costly. Mitigating solutions often include finding sub-optimal paths by reducing the time horizon. Other methods, such as Multiple-shooting methods [3] and Rapidly-exploring Random Trees (RRT) [11, 12] should also be considered.

A path planner generally generates paths for its agents by trying to optimize an objective function, and the natural approach is to propose a function of the maps discussed in Section III-B by weighted summation. Conceptually, the local value can then be calculated as

$$
m(x)=\sum_{i} w_{i}(x) m_{i}(x) .
$$

for maps $m_{i}(x)$ (themselves functions of respective inputs, such as previous amount of information in an area) and weights $w_{i}(x)$ evaluated at position $x$.

This equation yields a two-dimensional landscape exemplified in Figure 4 with estimated velocity covariance in ice flows - which can be navigated to find an optimal path. Note that actually following the path will yield information which possibly warrants re-planning.

Further, note that (1) implies that to form a twodimensional map $\{m(x)\}_{x \in X}$ over region $X$, information is only needed from the individual maps $\left\{m_{i}(x)\right\}_{x \in X}$, i.e. the cut-outs from the same region of each map. This means that we can partition the map into independent parts, for example assigning different areas to different groups of agents.

Besides from optimizing the objective function, the optimization routine should also take into account the 
limitations of the problem, such as maximum total (remaining) range of an agent. In the path-planning, multiple agents can be planned for simultaneously, to collaborate towards a common goal.

The path-planning described in this section constitutes future work in terms of the implementation of the system of Figure 1.

\section{Results}

Partial results of the different subsystems have been published previously in [17-19], but are recapitulated here as a descriptive example of the accomplished work.

In $[17,19]$, we introduced the spatially indexed Labeled Multi-Bernoulli (LMB) filter for scalable tracking of sea ice. The LMB implementation was used to track the ice movements in Kongsfjorden, Svalbard, over a period of seven hours, with scans delivered every three minutes. In Figure 3, we see the tracks of tracked ice objects build up over time.

The stationary sea ice, shown in green, changes only slightly over the course of the experiment, suggesting it would remain largely undetected if treated as drift ice. The detections of drift ice, shown in red, suffer many false alarms, but the LMB filter manages to confirm the targets, shown as ellipses, and maintain their tracks, shown as lines, over large stretches of water.

In [18], these results were expanded upon to generate Gaussian field-maps of the estimated winds and currents, as in Figure 4a.

\section{Conclusion}

In this paper we discuss the high-level design of an ice management system capable of feeding back the information it collects to the optimization of paths for future data acquisition. The principle is based on a feedback loop with high-level paths as the interface to the moving agents. Since all the components of the proposed system can be partitioned, it is believed to be well suited for scaling up to the geographical expanse that is the Arctic. Further, a standardized sensor interface is discussed to accomodate a range of available sensors. While pathplanning has yet to be implemented to close the loop in a true system, several algorithms have been mentioned - and several others exist — to solve the multi-agent planning problem at hand.

In previous papers, the implementation of various of the other subsystems have been discussed, and in this paper we wish to put them all into context and perspective of the greater goal. In continued work, we expect to move further towards closing the loop through further consideration of scalable metrics for information quality, as well as applying path-planning algorithms to the posed problem in a scaled-up scenario.

\section{ACKNOWLEDGMENTS}

This project has received funding from the European Union's Horizon 2020 research and innovation programme under the Marie Skłodowska-Curie grant agreement No 642153, as well as the Research Council of Norway through the Centres of Excellence funding scheme, grant number 223254 - NTNU-AMOS. The project has also been supported by funding from Vinnova Industry Excellence Center LINK-SIC, the Swedish strategic research center Security Link.

\section{REFERENCES}

[1] "International Ice Patrol." [Online]. Available: https: //www.navcen.uscg.gov/?pageName=IIPHome

[2] A. Albert, F. S. Leira, and L. Imsland, "UAV Path Planning using MILP with Experiments," Modeling, Identification and Control, vol. 38, no. 1, pp. 21-32, 2017.

[3] H. G. Bock and K. J. Plitt, "A multiple shooting algorithm for direct solution optimal control problems," in Proceedings of the 9th IFAC world congress. Budapest: Pergamon Press, 1984, pp. 243-247.

[4] K. Eik, "Review of Experiences within Ice and Iceberg Management," Journal of Navigation, vol. 61, no. 04, p. 557, 2008.

[5] F. Gustafsson, Statistical Sensor Fusion. Studentlitteratur, 2010.

[6] M. Hals and M. Skjønhaug, "Optimization of Coordinated Path Planning for Autonomous Vehicles in Ice Management," MSc. thesis, Norwegian University of Science and Technology, 2017.

[7] J. Haugen, "Autonomous Aerial Ice Observation," Ph.D. dissertation, Norwegian University of Science and Technology, 2014.

[8] J. Hnatiuk, "Exploration Methods in the Canadian Arctic," Cold Regions Science and Technology, vol. 7, pp. 181-193, 1983.

[9] M.-K. Hu, "Visual pattern recognition by moment invariants," IRE Transactions on Information Theory, vol. 8, pp. 179-187, 1962.

[10] S. M. Langeveld, "Unmanned Aerial Vehicle Mission Planning for Combined Iceberg Detection and Tracking Missions," MSc. thesis, Norwegian University of Science and Technology, 2017.

[11] S. M. LaValle, "Rapidly-Exploring Random Trees: A New Tool for Path Planning," Tech. Rep., 1998.

[12] —, Planning Algorithms. New York, NY, USA: Cambridge University Press, 2006.

[13] F. S. Leira, T. A. Johansen, and T. I. Fossen, "A UAV Ice Tracking Framework for Autonomous Sea Ice Management."

[14] F. S. Leira, "Object Detection and Tracking With UAVs," Ph.D. dissertation, Norwegian University of Science and Technology, 2017. 
[15] K. Moran, J. Backman, and J. W. Farrell, "Deepwater drilling in the Arctic Ocean's permanent sea ice," vol. 302, pp. 1-13, 2006.

[16] J. Olofsson, E. Brekke, T. I. Fossen, and T. A. Johansen, "Spatially Indexed Clustering for Scalable Tracking of Remotely Sensed Drift Ice," in IEEE Aerospace Conference Proceedings, Big Sky, MT, USA, 2017.

[17] J. Olofsson, E. Brekke, and T. A. Johansen, "Cooperative Remote Sensing of Ice using a Spatially Indexed Labeled Multi-Bernoulli Filter," in Int. Conf. Unmanned Aircraft Systems, Miami, 2017.

[18] J. Olofsson, A. L. Flåten, C. Veibäck, and T. R. Lauknes, "Gaussian Field Current Estimation from Drift Sea Ice Tracking with the Labeled MultiBernoulli Filter," in Proceedings of OCEANS 2017 MTS/IEEE, Anchorage, 2017.

[19] J. Olofsson, C. Veibäck, and G. Hendeby, "Sea Ice Tracking with a Spatially Indexed Labeled Multi-Bernoulli Filter," in Information Fusion (FUSION), 2017 20th International Conference on, Xi'an, China, 2017.

[20] P. Skoglar, Tracking and Planning for Surveillance Applications, 2012, no. 1432.

[21] K. L. Sørensen, A. S. Helland, and T. A. Johansen, "Carbon nanomaterial-based wing temperature control system for in-flight anti-icing and de-icing of unmanned aerial vehicles," IEEE Aerospace Conference Proceedings, vol. 2015-June, 2015.

[22] B. Wright, "Full Scale Experience with Kulluk Stationkeeping Operations in Pack Ice (With Reference to Grand Banks Developments)," Tech. Rep., 2000. 